\title{
EL GÉNERO OXYPETALUM (APOCYNACEAE) EN URUGUAY,NUEVA ESPECIE Y CLAVE DE IDENTIFICACIÓN
}

\author{
Andrés González¹ \& Héctor A. Keller²,3
}

\begin{abstract}
${ }^{1}$ Departamento de Botánica, Museo Nacional de Historia Natural, 25 de mayo 582, CP 11000, Montevideo, Uruguay. ${ }^{2}$ Instituto de Botánica del Nordeste, IBONE-CONICET, Sargento Juan Bautista Cabral 2131, CC 209, 3400 Corrientes, Corrientes, Argentina; ibone@agr.unne.edu.ar (autor corresponsal).

${ }^{3}$ Facultad de Ciencias Forestales, Universidad Nacional de Misiones, Bertoni 124 km 3, 3380 Eldorado, Misiones, Argentina.
\end{abstract}

Abstract. González, A. \& H. A. Keller. 2020. The genus Oxypetalum (Apocynaceae) in Uruguay, a new species and a key for identification. Darwiniana, nueva serie 8(1): 318-327.

Based on herbarium material collected in Rivera, Uruguay, a species new to science, Oxypetalum brussae H.A. Keller \& A. González is described and illustrated here. The species is similar in its vegetative and floral morphology to Oxypetalum teyucuarense Farinaccio \& H.A. Keller. The new species allows the list of taxa of the genus for Uruguay to be increased to 19, three of which are endemic to this country.

Keywords. Asclepiadoideae; new taxon; Oxypetalinae; taxonomy.

Resumen. González, A. \& H. A. Keller. 2020. El género Oxypetalum (Apocynaceae) en Uruguay, nueva especie y clave de identificación. Darwiniana, nueva serie 8(1): 318-327.

Sobre la base de muestras recolectadas en Rivera, Uruguay, se describe e ilustra Oxypetalum brussae H.A. Keller \& A. González, una especie nueva para la ciencia. La especie es similar en su morfología vegetativa y floral a Oxypetalum teyucuarense Farinaccio \& H.A. Keller. La nueva especie permite elevar a 19 la lista de taxones del género para Uruguay, tres de los cuales son endémicos del país.

Palabras clave. Asclepiadoideae; nuevo taxón; Oxypetalinae; taxonomía.

\section{INTRODUCCIÓN}

Oxypetalum R. Br. constituye uno de los dos géneros de mayor riqueza específica entre las Apocynaceae - Asclepiadoideae del Nuevo Mundo, con más de 130 taxones (Endress et al., 2018). Estas especies se encuentran distribuidas desde el centro de la Argentina hasta México, pero con mayor diversidad en la Argentina, Brasil y Paraguay (Meyer,
1943; Farinaccio, 2004; Fontella Pereira et al., 2004, 2010; Marquete et al., 2007; Ezcurra et al., 2008). El número de representantes del género se ha incrementado en la última década, sobre todo debido a nuevas combinaciones y restablecimientos nomenclaturales (Rapini et al., 2011; Liede \& Meve, 2015) y al hallazgo de nuevos representantes, principalmente en el Cono Sur de América del Sur (Farinaccio \& Keller, 2014; Keller, 2015, 2020; Keller \& Funez, 2017; 
Martín et al., 2017; González et al., 2018). Para Uruguay se han mencionado un total de 17 especies (Ezcurra et al., 2008, González et al., 2018; Zuloaga et al., 2019).

Recientes campañas de recolección efectuadas en el Paisaje Protegido Valle del Lunarejo, en el departamento de Rivera, Uruguay, en el marco del relevamiento de la flora de Uruguay, permitieron hallar una población de una especie de Oxypetalum R. Br. cuya combinación de caracteres no se ajusta a ningún taxón descripto hasta el presente. Su descripción, sumada a la inminente resurrección de Oxypetalum megapotamicum Spreng. (Keller et al., en prensa), elevan la riqueza de especies de Oxypetalum de Uruguay a 19 taxones, de los cuales solo cuatro se constituyen de plantas volubles trepadoras, siendo todas las demás erectas o decumbentes. En la presente contribución se describe e ilustra la nueva especie, además todas las especies hasta el presente registradas para el Uruguay se incluyen en una clave y son ilustradas mediante fotografías.

\section{MATERIALES Y MÉTODOS}

En el herbario CTES se estudiaron muestras de Oxypetalum teyucuarense Farinaccio \& H. A. Keller y otras especies afines. Para obtener las imágenes empleadas en el proceso de estudio del material y para confeccionar las ilustraciones correspondientes a la especie que aquí se describe se utilizaron una cámara fotográfica con alta resolución (Sonny DSC-HX400V) y un microscopio estereoscópico (Zeizz Stemi DV4). $\mathrm{El}$ indumento y el polinario fueron examinados con microscopio óptico (Leica DM500) con cámara fotográfica incorporada (ICC50). Para extraer los polinarios y estudiar los caracteres reproductivos, las flores fueron ablandadas mediante su decocción en agua a $100^{\circ} \mathrm{C}$, correspondiendo por lo tanto las dimensiones descriptas al material rehidratado.

La terminología botánica empleada sigue a la obra de Font Quer (1993). La diagnosis se redactó en latín expresando en el menor espacio posible los caracteres diferenciales de la especie (Stearn, 2006).

\section{RESULTADOS}

Oxypetalum brussae H.A. Keller \& A. González sp. nov. TIPO: Uruguay, Rivera, Paisaje Protegido Valle del Lunarejo, Bajada de Pena. 20/01/2020, fl, fr, Keller \& González 14079 (holotipo CTES!). Figs. 1 y 2.

Oxypetalum teyucuarense Farinaccio \& H.A. Keller affinis sed coronae lobulis tridentatis et retinaculo breviore.

Sufrútice latescente, vástagos erectos de hasta $120 \mathrm{~cm}$ de altura; tallos cilíndricos de 1,5-2 mm de diám., entrenudos glabrescentes en la base, pubescentes hacia el ápice y en nudos basales, tricomas 2-4 septados, uniseriados, blanquecinos, patentes o adpreso-curvados, de 0,1-0,3 mm de long., con superficie cubierta de pequeñas prominencias elípticas; entrenudos de 1,7-17 cm de long. Hojas opuestas; pecíolos de 3-10 $\mathrm{mm}$, con pubescencia similar a la de los nudos; láminas lineares, 4-9 x 0,1-0,7 cm, concoloras, epifilo pubescente con tricomas cortos, 2-3 septados, hipofilo pubescente con numerosos tricomas cortos y en menor cantidad largos pluricelulares; margen entero, base redondeada a cordulada con 1-2 coléteres digitiformes de 0,3-0,5 mm en la cara adaxial, ápice agudo, venación broquidódroma con 9-15 pares de nervaduras secundarias. Inflorescencias extra-axilares umbeliformes, erectas, alternas, con 2-6 flores; pedúnculos de 1,5-7 x 0,6-1 mm, pubescentes; brácteas filiformes a lineares, pubescentes, de 1,5-3 x 0,2-0,5 mm; pedicelos vinosos de 1,5-6 x $0,5 \mathrm{~mm}$ Cáliz dividido hasta cerca de la base; tubo de 0,3-0,35 mm de alt. x 0,9-1,1 $\mathrm{mm}$ de diám.; lóbulos lanceolados, agudos, de 3-3,2 x 0,9-1,1 mm, pubescentes en ambas caras, con 3-4 coléteres digitiformes de 0,25-0,3 mm en el seno, del lado interno. Corola con tubo campanulado, 2,8-3 $\mathrm{mm}$ de alt. x 3-4 mm de diám., superficie externa pubescente, lóbulos verde-amarillentos, oblongo-ovados 7-7,2 x 2,6-2,7 mm, retorcidos, pubescentes abajo, glabros arriba. Corona crema o lilácea; lóbulos lanceolados de 4,2-4,4 x 1,8-1,9 mm, soldados a la base del ginostegio, divididos 


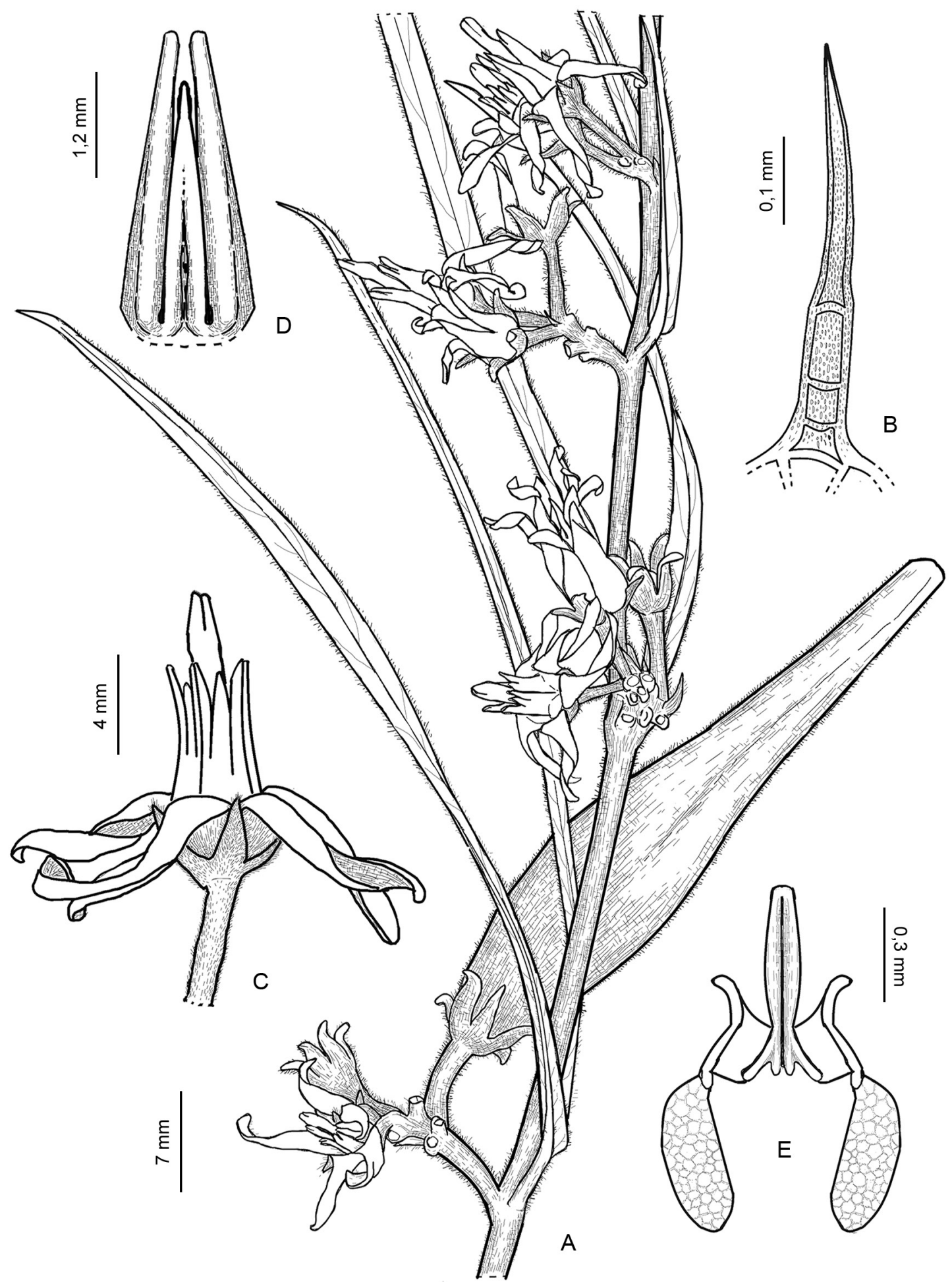

Fig. 1. Oxypetalum brussae. A, rama florífera. B, tricoma de la hoja. C, flor. D, pieza de la corona en cara ventral. E, polinario. (Dibujos de Héctor A. Keller, del Holotipo). 


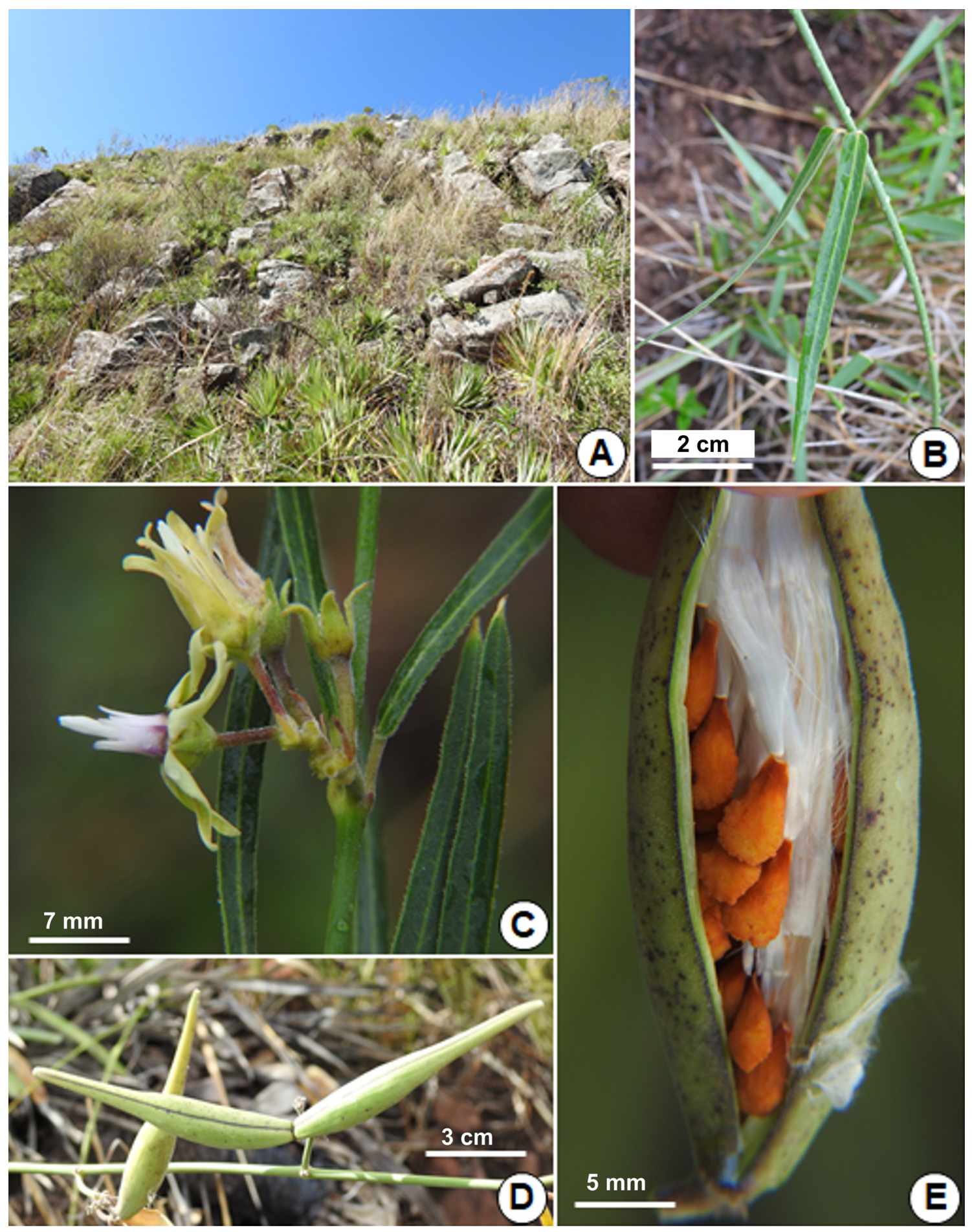

Fig. 2. Oxypetalum brussae. A, ambiente. B, hojas basales. C, flores. D, frutos simples y geminados. E, semillas en el fruto. (Fotos de Andrés González). Figura en color en la versión en línea http://www.ojs.darwin.edu.ar/ index.php/darwiniana/article/view/882/1180 
en el ápice en dos dientes laterales de 1,8-2 x 0,25-0,3 mm, y un diente interno de 0,9-1 x $0,3-0,35 \mathrm{~mm}$ del cual se proyectan dos costas ventrales hasta la base del lóbulo. Ginostegio sésil. Anteras 0,8-0,9 x 0,65-0,75 mm, membrana apical ovada de 0,95-1 x 0,8$0,9 \mathrm{~mm}$. Retináculo oblongo-oblanceolado, 0,50-0,51 x 0,09-0,1 mm, ápice truncado, caudículas traslúcidas de 0,12-0,14 x 0,18$0,19 \mathrm{~mm}$ con espesamientos opacos en la base y en el borde externo, este último rematando en un diente curvo, exerto de ca. $0,12 \mathrm{~mm}$, polinios reniformes de 0,39-0,4 x 0,15-0,16 mm. Cabeza estilar pentalobulada, convexa, de 0,5-0,6 x 0,95-1 mm, apéndice estigmático blanco, carnoso, exerto, de 6-6,5 $\mathrm{x}$ 0,8-1 $\mathrm{mm}$, bifurcado en el tercio distal en dos ramas crasas, conniventes y algo retorcidas, de 1,9-2 x 0,5-0,6 mm, porción basal indivisa de 2,8-3,1 x 0,8-1 mm. Ovario dialicarpelar ca. $1,8 \times 1,5 \mathrm{~mm}$. Folículos verdeamarillentos, fusiformes, agudos, rectos o algo curvados, de 7-10 x 1-1,5 cm, con frecuencia geminados en cuyo caso son divergentes en un ángulo de más de $130^{\circ}$; semillas obovadas 5-6,5 x 2-3 mm, borde sinuoso-dentado, superficie rugosa, con penacho de 2,5-4 mm de longitud.

Etimología. Especie dedicada al Ingeniero Agrónomo Carlos Antonio Brussa, exdirector del Museo y Jardín Botánico "Prof. Atilio Lombardo" y profesor de la Facultad de Agronomía de la Universidad de la República en Montevideo, Uruguay, quien ha contribuido al conocimiento de la flora arbórea y arbustiva del país, así como transmitió con generosidad sus conocimientos a las generaciones futuras.

Distribución y hábitat. Oxypetalum brussae crece sobre laderas de arenisca y entre rocas de basalto a elevaciones entre los 260-280 m s. m., en cerros que constituyen la Cuchilla de Haedo en el departamento de Rivera, al norte de Uruguay, compartiendo hábitat con una comunidad herbáceo-arbustiva dominada por Dyckia sp. (Bromeliaceae), Parodia herteri (Werderm.) N.P. Taylor (Cactaceae), Oxypetalum coeruleum (D. Don) Decne.
(Apocynaceae), Calea clematidea Baker (Asteraceae) y Acalypha communis Müll. Arg. (Euphorbiaceae), en un área restringida de aproximadamente $4000 \mathrm{~m}^{2}$.

Fenología y estado de conservación. Florece en diciembre-febrero, fructifica en enero-marzo. Según los criterios de IUCN (2019), la especie puede ser considerada en Peligro Crítico (CR-B2[a]) debido a que sólo se conoce una población en una pequeña área que en la actualidad se encuentra incluida en el Sistema Nacional de Áreas Protegidas de Uruguay. Las principales amenazas que presenta la especie es el pisoteo y consumo por parte de ganado, así como el efecto de incendios intencionales con consecuencias desconocidas sobre la población. Después de Oxypetalum nigricans (Decne.) Liede \& Meve y $O$. marchesii C. Ezcurra \& A. González, $O$. brussae H.A. Keller \& A. González es la tercera especie del género endémica de Uruguay.

Observaciones taxonómicas. La especie es morfológicamente afín a $O$. teyucuarense Farinaccio \& H.A. Keller, y aunque distantes geográficamente, crecen en el mismo tipo de ambiente, campos rupestres con vegetación abierta sobre elevaciones de arenisca. Comparten el hábito grácil, la pubescencia, las hojas lineares laxamente dispuestas, las inflorescencias cortamente pedunculadas y paucifloras, los lóbulos del cáliz con pubescencia en ambas caras. La corola tiene lóbulos reflexos, verde amarillentos en $O$. brussae, mientras que en $O$. teyucuarense son erectos con extremos recurvos y de color marrón-rojizos con tonos púrpuras. En ambos taxones el apéndice estigmático es exerto y con ramas gruesas y crasas, pero en $O$. teyucuarense estas ramas son ruminadas e incluso más gruesas que el pie que las sostiene. Los polinarios son morfológicamente similares, aunque el retináculo es sensiblemente más pequeño en $O$. brussae. La diferencia más sustancial está en los lóbulos de la corona tridentados de O. brussae, un atributo que además permite distinguirla dentro de los cerca de veinte taxones del género con hojas lineales. 


\section{Las especies de Oxypetalum que han sido registradas para Uruguay se pueden diferenciar mediante la siguiente clave.}

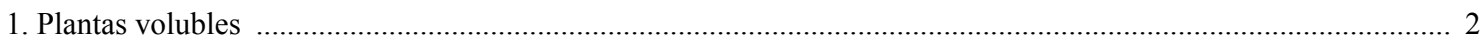

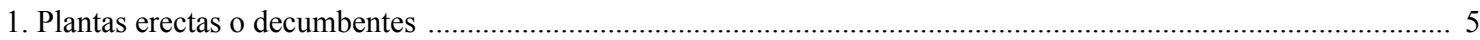

2(1). Cabeza estilar mútica ………………………..................................... O. megapotamicum Spreng. (Fig. 3A)

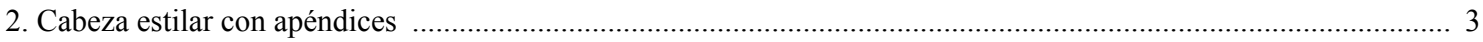

3(2). Apéndice estigmático con 5-7 ramas ............................... O. sylvestre (Hook. \& Arn.) Goyder \& Rapini (Fig. 3B)

3. Apéndice estigmático bifurcado

4(3). Caudículas dentadas

O. pannosum Decne. var. pannosum (Fig. 3C)

4. Caudículas edentadas

O. macrolepis (Hook. \& Arn.) Decne. (Fig. 3D)

5(1). Lóbulos de la corona con dos dientes laterales externos y un diente central interno .......................................... 6

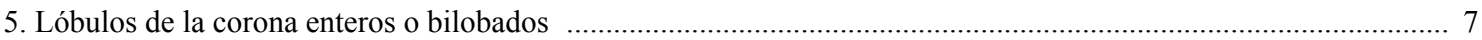

6(5). Hojas lineares, caudículas dentadas

O. brussae H.A. Keller \& A. González

6. Hojas ovadas, caudículas edentadas

O. commersonianum (Decne.) Fontella \& E.A. Schwarz (Fig. 3E)

7(5). Flores amarillo-doradas, ramas del apéndice estigmático filiformes, divergentes, con extremos incurvos

O. aurantiacum Malme ex Chodat \& Hassl. (Fig. 3F)

7. Flores de otros colores, ramas del apéndice estigmático cuando filiformes conniventes o divergentes con extremos

recurvos

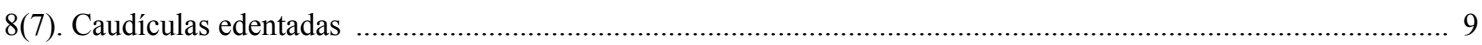

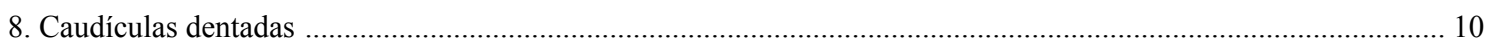

9(8). Flores rosadas, lilas o rojas, ramas del apéndice estigmático filiformes ............... O. coccineum Griseb. (Fig. 4A)

9. Flores moradas, ramas del apéndice estigmático cónicas ................. O. nigricans (Decne.) Liede \& Meve (Fig. 4B) 10(8). Láminas foliares de menos de $2 \mathrm{~cm}$ long. .......................................... O. microphyllum Hook. \& Arn. (Fig. 4C)

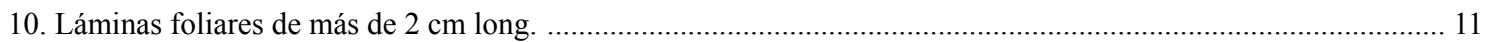

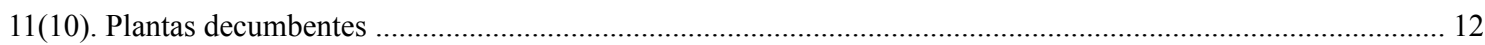

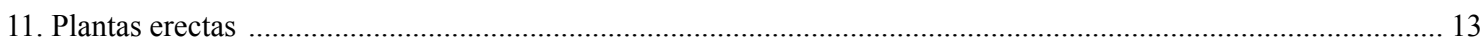

12(11). Apéndice estigmático incluso entre los lóbulos de la corona ....... O. marchesii C. Ezcurra \& A. González (Fig.4D)

12. Apéndice estigmático exerto ............................... O. tomentosum Wight ex Hook. \& Arn. var. tomentosum (Fig. 4E)

13(11). Láminas foliares lineares, cerca de 20 veces más largas que anchas ..................... O. schulzii Malme (Fig. 4F)

13. Largo de la lámina foliar menos de 10 veces el ancho

14(13). Flores moradas, piezas de la corona enteras o con dos lóbulos superficiales redondeados

O. arnottianum H. Buek (Fig. 5A)

14. Flores celestes, verdosas, blanquecinas, rosadas, lilas o con tonos rojizos, nunca moradas, piezas de la corona con dientes o lóbulos agudos

15(14). Flores celestes

O. coeruleum (D. Don) Decne. (Fig. 5B)

15. Flores verdosas, blanquecinas, rosadas, lilas o con tonos rojizos, nunca celestes

16(15). Corola con lóbulos retrorso-plegados sobre sí mismos desde el tercio basal. Apéndice estigmático con ápice carnoso obcónico de más de 1,5 mm diám.

O. capitatum Mart. subsp. capitatum (Fig. 5C)

16. Corola con lóbulos patentes o reflexos, pero no plegados desde el tercio basal, apéndice estigmático con ápice cilíndrico o cónico

17(16). Corola con lóbulos patentes. Retináculo más largo que las polinias O. solanoides Hook. \& Arn. (Fig. 5D)

17. Corola con lóbulos reflexos. Retináculo más corto que las polinias

18(17). Corola con lóbulos lanceolados a linear-lanceolados $5-7$ x ca. $1,5 \mathrm{~mm}$

O. crispum Wight ex Hook. \& Arn. (Fig. 5E)

18. Corola con lóbulos filiformes retorcidos, $10-12 \mathrm{x}$ ca. $1 \mathrm{~mm}$

O. parviflorum (Decne.) Decne. var. parviflorum (Fig. 5F) 


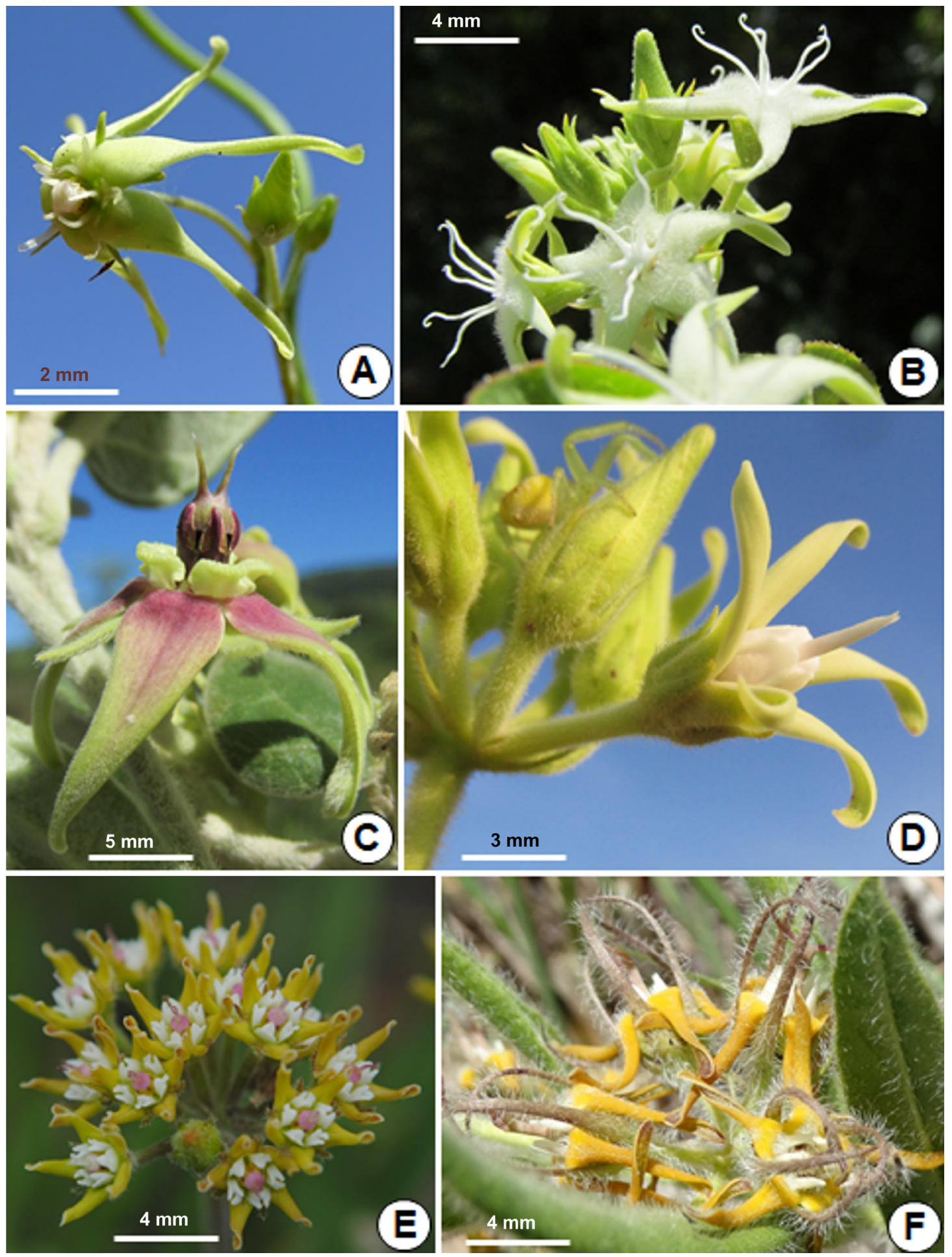

Fig. 3. A, Oxypetalum megapotamicum. B, O. sylvestre. C, O. pannosum var. pannosum. D, O. macrolepis. E, O. commersonianum. F, O. aurantiacum. (Fotos A-D: Héctor A. Keller; E: Andrés González; F: Leonardo Deble). Figura en color en la versión en línea http://www.ojs.darwin.edu.ar/index.php/darwiniana/article/view/882/1180 

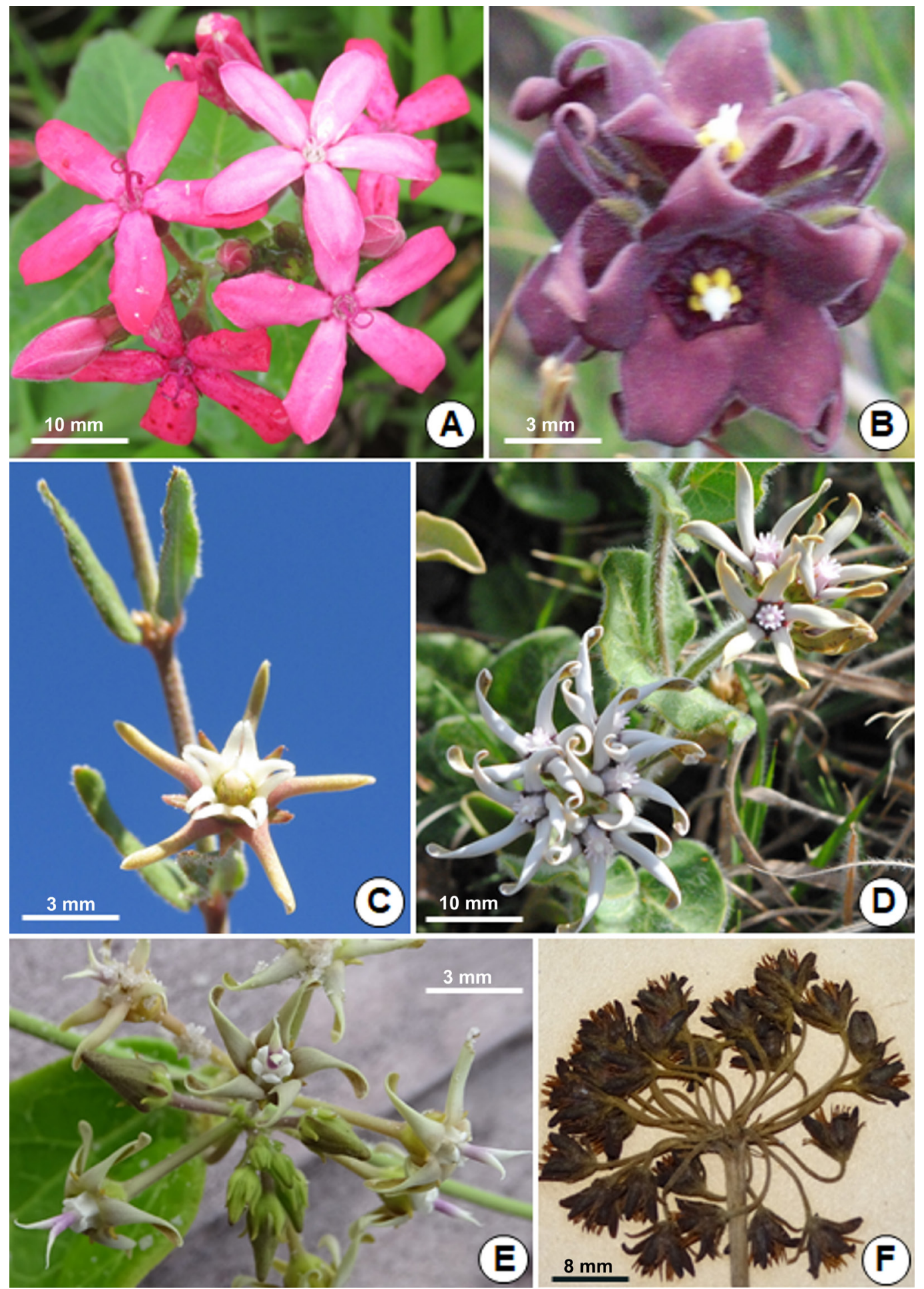

Fig. 4. A, Oxypetalum coccineum. B, O. nigricans. C, O. microphyllum. D, O. marchesii. E, O. tomentosum var. tomentosum. F, O. schulzii. (Fotos A, C, E, F: Héctor A. Keller; B, D: Andrés González). Figura en color en la versión en línea http://www.ojs.darwin.edu.ar/index.php/darwiniana/article/view/882/1180 

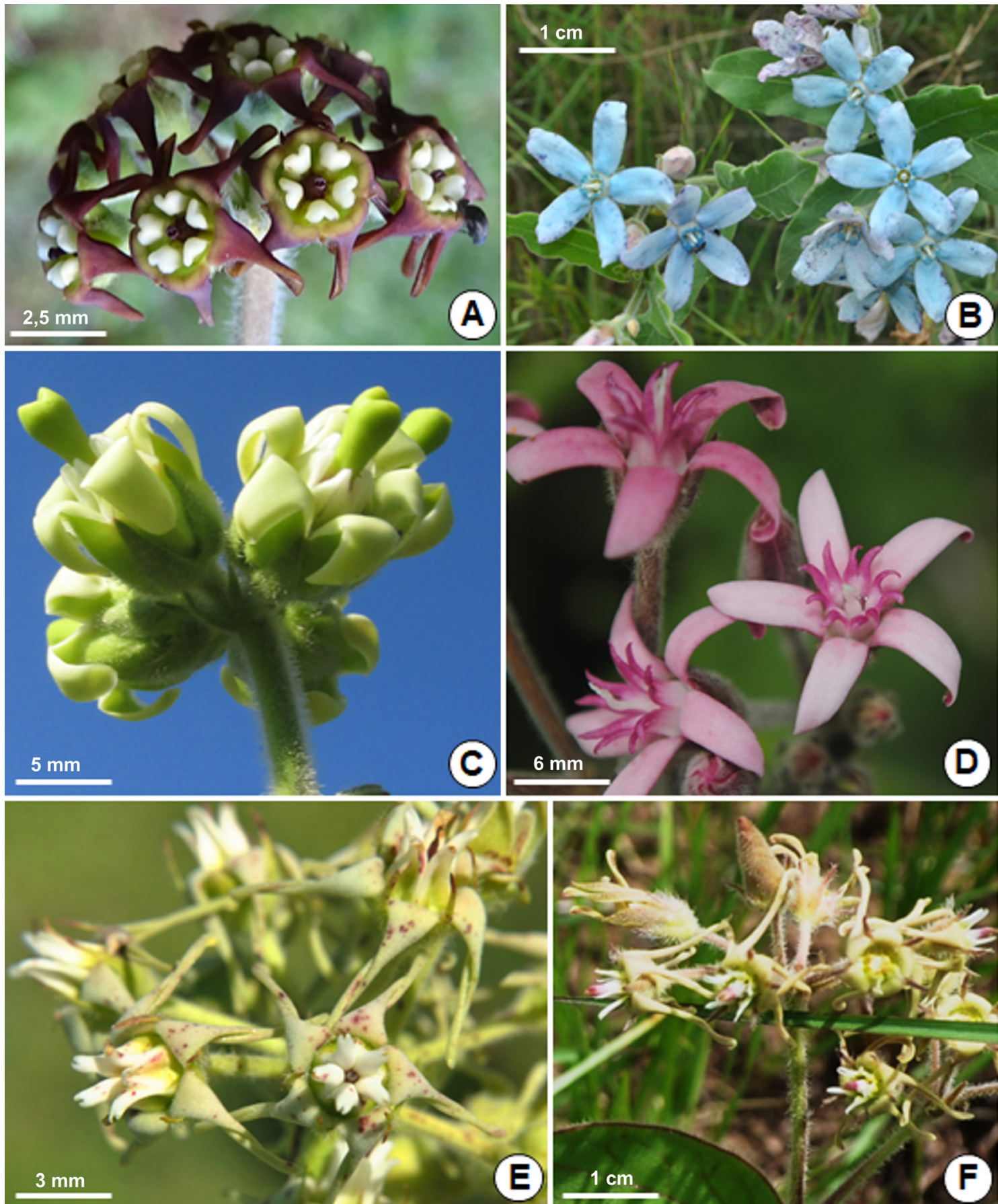

Fig. 5. A, Oxypetalum arnottianum. B, O. coeruleum. C, O. capitatum supsp. capitatum. D, O. solanoides. E, O. crispum. F, O. parviflorum var. parviflorum. (Fotos A, C: Héctor A. Keller; B, D, E: Andrés González; F: Cleusa Vogel). Figura en color en la versión en línea http://www.ojs.darwin.edu.ar/index.php/darwiniana/ article/view/882/1180 


\section{A. GONZÁLEZ \& H. A. KELLER. Oxypetalum en Uruguay}

\section{AGRADECIMIENTOS}

Se agradece a Naldo Pellizer de la Facultad de Ciencias Forestales UNaM por las microfotografías del polinario y tricomas. A Leonardo Deble (UNIPAMPA) por la foto de Oxypetalum aurantiacum y a Cleusa Vogel (UFRGS) por la foto de Oxypetalum parviflorum. Por último, agradecemos a los editores de la revista y a dos revisores anónimos por la valiosa contribución recibida para mejorar este artículo.

\section{BIBLIOGRAFÍA}

Endress M. E.; U. Meve, D. J. Middleton \& S. Liede-Schumann. 2018. Apocynaceae. En: Kadereit, J. W. \& V. Bittrich (eds.). The Families and Genera of Vascular Plants. Vol. 15. Flowering Plants, Eudicots, Apiales, Gentianales (excl. Rubiaceae). Springer; Heidelberg, New York, pp. 207-411.

Ezcurra, C.; J. Fontella Pereyra \& L. Kinoshita. 2008. Apocynaceae (incl. Asclepiadaceae), en Zuloaga, F. O.; O. Morrone \& M. Belgrano (eds.), Catálogo de las Plantas Vasculares del Cono Sur, vol. 2. Monographs in Systematic Botany from the Missouri Botanical Garden 107: 1090-1143. Farinaccio, M. A. 2004. Oxypetalum R. Br., en Wanderley, M. G. L.; G. L. Shepherd, T. S. Melhem \& A. M. Giulietti (eds.), Flora fanerogâmica do Estado de São Paulo 4, pp. 130-150, Fapesp/RiMa, São Paulo.

Farinaccio, M. A. \& H. A. Keller. 2014. Novelties in Oxypetalum (Apocynaceae-Asclepiadoideae) for the Argentine flora. Phytotaxa 184: 109-114.

Fontella Pereira, J.; M. C. Valente, N. M. S. Marquete \& C. L. F. Ichaso. 2004. Apocináceas, Asclepiadaóideas, en Reis, A. (ed.), Flora Ilustrada Catarinense. Herbário Barbosa Rodrigues, Itajaí, 1-250 pp.

Fontella Pereira, J.; S. A. Cáceres Moral \& M. B. de Goes. 2010. Sinopsis y tipificaciones de las especies del género Oxypetalum R. Br. (Asclepiadaceae) en Paraguay. Notula ad Floram paraquaiensem 106. Candollea 65: 394-402.

Font Quer, P. 1993. Diccionario de Botánica. 1244 pp. Ed. Labor, Barcelona.
González, A.; M. Fernández \& C. Ezcurra. 2018. Oxypetalum marchesii (Apocynaceae, Asclepiadoideae), an Endemic New Species from Uruguay. Systematic Botany 43: 812-817.

IUCN Standards and Petitions Subcommittee. 2019. Guidelines for Using the IUCN Red List Categories and Criteria. Version 14. Prepared by the Standards and Petitions Subcommittee. Disponible en www.iucnredlist. org/documents/RedListGuidelines.pdf

Keller, H. A. 2015. Oxypetalum radinsii (Apocynaceae: Asclepiadoideae), una nueva especie endémica de Misiones, Argentina. Bonplandia 24: 51-56.

Keller, H. A. 2020. Oxypetalum (Apocynaceae) en la Argentina: una nueva especie, nuevos sinónimos y una clave de identificación. Bonplandia 29: 81-99.

Keller, H. A. \& L. A. Funez. 2017. Una nueva especie de Oxypetalum (Apocynaceae: Asclepiadoideae) de Santa Catarina, Brasil. Bonplandia 26: 119-124.

Keller, H. A.; L. A. Funez \& S. Liede-Schumann. (En prensa). Novelties in Oxypetalum (Apocynaceae: Asclepiadoideae): a new species and revalidation of the name $O$. megapotamicum. Rodriguesia 71.

Liede, S. \& U. Meve. 2015. Synonymy of three South American genera in Apocynaceae, and new combinations in Oxypetalum and Tassadia. Phytotaxa 202: 35-44.

Marquete, N. F. S.; J. Fontella Pereira \& M. C. Valente. 2007. Asclepiadoideae (Apocynaceae) from southeastern Brazil. I. The genus Oxypetalum from Rio de Janeiro State, Brazil. Annals of the Missouri Botanical Garden. 94: 435-462.

Martín, C. M.; C. A. Zanotti \& C. Ezcurra. 2017. A New Species of Oxypetalum (Apocynaceae, Asclepiadoideae) from Northern Argentina. Systematic Botany 42: 578-583.

Meyer, T. 1943. Revisión de las especies argentinas del género Oxypetalum (Asclepiadaceae). Lilloa 9: 5-72.

Rapini, A.; J. Fontella Pereira \& D. J. Goyder. 2011. Towards a stable generic circumscription in Oxypetalinae (Apocynaceae). Phytotaxa 26: 9-16.

Stearn, W. 2006. Latín Botánico, historia, gramática, sintaxis, terminología y vocabulario. Ed. Omega, Barcelona, 644 pp.

Zuloaga, F. O.; M. J. Belgrano \& C. A. Zanotti. 2019. Actualización del Catálogo de las Plantas Vasculares del Cono Sur. Darwiniana, nueva serie 7(2): 208-278. 